\title{
Performance Analysis of NLFM Signals with Doppler Effect and Background Noise
}

\author{
Nettem Adithya Valli, Daniel Elizabath Rani, Chandu Kavitha
}

\begin{abstract}
This paper focuses on the study of effect of background noise and Doppler Effect on various Nonlinear Frequency Modulation (NLFM) waveforms designed using two stage piece-wise and three stage piece-wise linear and non linear functions. The background noise investigated is Additive White Gaussian Noise (AWGN). Simulations are carried out for different target speeds ranging from 100 to $5000 \mathrm{~km} / \mathrm{hour}$ to study Doppler Effect. The simulations are carried out using Matlab software. Among the waveforms designed, the NLFM function designed using two piece-wise Linear Frequency Modulation (LFM) is observed to be Doppler tolerant and also not affected by noise, as the SNR changes from -20 to $20 \mathrm{~dB}$ the peak side-lobe level (PSL) of this signal is around $-34.84 \mathrm{~dB}$.
\end{abstract}

Keywords: Linear frequency modulation, Non-Linear frequency modulation, Piece-wise functions, Peak-sidelobe level, Doppler Tolerance.

\section{INTRODUCTION}

Non-Linear frequency modulation (NLFM) method suppresses side-lobe levels better than LFM for better resolution and detectability in radar surveillance applications. As the parameters PSL and main lobe width (MLW) affect the performance of detectability and resolution respectively, several NLFM waveforms have been investigated. Not all the waveforms can provide sufficient reduction in side-lobes [1], [2]. NLFM signals design available so far is generally classified in 2 directions. First classification is design by using desired PSD function shape by means of different methods such as iterative methods and stationary phase principle method. Second is to design a NLFM signal using piece-wise LFM signals [3]. Many studies attempted to design most favourable (less side-lobe) NLFM signals [4-6]. Authors in [7] proved that a pulse with higher FM rates at the starting point of pulse and the ending point of pulse can reduce side-lobe values better than LFM, as higher FM rate portions reduce the Fresnel ripples in the waveform spectrum. Authors in [8] and [9] explained this technique through the piece-wise NLFM waveforms. They explained that if the higher FM rate portions are chosen correctly, side-lobes will decline. Majority of approaches discussed piece-wise LFM and NLFM waveforms are demonstrated. The present study primarily focuses on NLFM waveform design by combining piece-wise linear and non-linear functions in such a way that the subsequent function is capable of generating an overall NLFM signal.

Revised Manuscript Received on February 05, 2020.

* Correspondence Author

C. Kavitha*, Department of Physics, Gitam University, Vishakhapatnam, India. E-mail: kavitha.chandu@gitam.edu

N. Adithya valli, assistant professor, Lingaya's engineering college Vijayawada, India.

D. Elizabath rani, Professor \& Principal, GITAM Institute of Technology, GITAM University, Visakhapatnam, India.

(C) The Authors. Published by Blue Eyes Intelligence Engineering and Sciences Publication (BEIESP). This is an open access article under the CC BY-NC-ND license (http://creativecommons.org/licenses/by-nc-nd/4.0/)
In two and three stage NLFM signals, LFM signals with different sweep rates is considered where as in modified NLFM waveforms instead of using LFM in both segments, either one Or both the segments is modified using an exponential function. Performance analysis of designed NLFM waveforms against Doppler Effect and background noise is carried out.

\section{NON-LINEAR FREQUENCY MODULATION} (NLFM)

All the NLFM signals are generated using LFM signals with different sweep rates, by changing the frequency linearly through the given time frame. The present study primarily concentrations on NLFM signal design by combining piecewise a linear and non-linear function so that the resultant functions are capable of generating an overall NLFM signal. In two and three stage NLFM, combination of LFM signals with different sweep rates is considered where as in modified NLFM waveforms instead of using LFM in both segments, either one or both of the segments is modified using an exponential function.

\section{A. Two-Stage NLFM (NLFM1)}

A Non-linear signal is generated using simple two-stage piece-wise LFM functions as shown below.
$f(t)=\left\{\begin{array}{c}\alpha_{0} t \\ B_{1}+\alpha_{1}\left(t-T_{1}\right)\end{array}\right.$
$0 \leq t \leq T_{1}$
$T_{1} \leq t \leq\left(T_{1}+T_{2}\right)$

Eq. (1) characterizes the instantaneous frequency function variation of NLFM signal formed by concatenating two piece-wise LFM functions with $\alpha_{0}$ and $\alpha_{1}$ being the sweep rate in the first and second stage. The pulse width of the chirp signal " $t$ " is divided into two time slots with individual pulse widths $T_{1}$ and $T_{2}$ and the corresponding bandwidths being $B_{1}$ and $B_{2}$, the equivalent sweep rates is given by following equation

$$
\alpha_{0}=\frac{B_{1}}{T_{1}}, \quad \alpha_{1}=\frac{B_{2}}{T_{2}}
$$

The phase variation of the above given NLFM signals can be derived by Eq. (1) and is given by Eq. (2)

$$
\begin{aligned}
& \varphi(t)=\int f(t)= \\
& \left\{\begin{array}{cc}
\alpha_{0} \frac{t^{2}}{2} & 0 \leq t \leq T_{1} \\
B_{1} t+\alpha_{1}\left(\frac{t^{2}}{2}-T_{1} t\right) & T_{1} \leq t \leq T_{1}+T_{2}
\end{array}\right.
\end{aligned}
$$




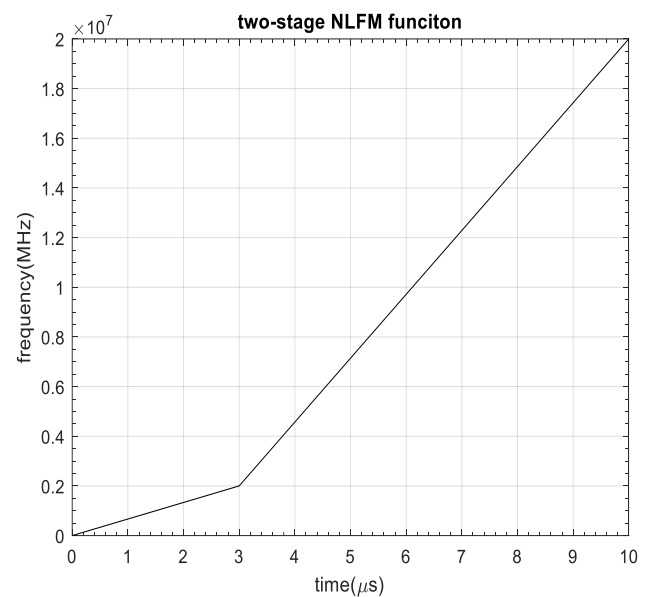

Figure.1 Two-stage NLFM function

\section{B. Three-Stage NLFM (NLFM2)}

Three-Stage NLFM signal is formed by concatenating the instantaneous frequency functions of three piece-wise LFM function with each different sweep rates. The instantaneous frequency of this NLFM is represented in Eq. (3)

$$
\begin{aligned}
& \mathbf{f}(\mathrm{t})= \\
& \left\{\begin{array}{cc}
\alpha_{0} t & 0 \leq t \leq T_{1} \\
B_{1}+\alpha_{1}\left(t-T_{1}\right) & T_{1} \leq t \leq\left(T_{1}+T_{2}\right) \\
B_{1}+B_{2}+\alpha_{2}\left(t-\left(T_{1}+T_{2}\right)\right. & \\
\left(T_{1}+T_{2}\right) \leq t \leq\left(T_{3}+\left(T_{1}+T_{2}\right)\right)
\end{array}\right\}
\end{aligned}
$$

and corresponding sweep rates are defined as follows

$$
\alpha_{0}=\frac{B_{1}}{T_{1}}, \quad \alpha_{1}=\frac{B_{2}}{T_{2}}, \quad \alpha_{2}=\frac{B_{3}}{T_{3}}
$$

The phase variation given in Eq. (4) of this concatenated NLFM function can be attained through integrating Eq. (3)

$$
\begin{aligned}
& \varphi(t)=\int f(t)= \\
& \left\{\begin{array}{cc}
\alpha_{0} \frac{t^{2}}{2} & 0 \leq t \leq T_{1} \\
B_{1} t+\alpha_{1}\left(\frac{t^{2}}{2}-T_{1} t\right) & T_{1} \leq t \leq T_{1}+T_{2} \\
B_{1} t+B_{2} t+\alpha_{2}\left(\frac{t^{2}}{2}-\left(T_{1}+T_{2}\right) t\right) & \left(T_{1}+T_{2}\right) \leq t \leq\left(T_{3}+\left(T_{1}+T_{2}\right)\right)
\end{array}\right.
\end{aligned}
$$

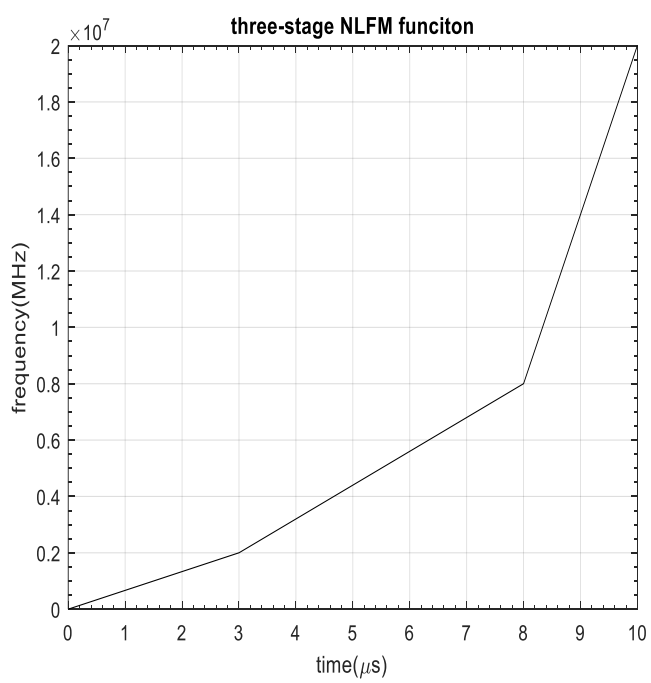

Figure.2 Three-stage NLFM function

\section{MODIFIED NLFM (MNLFM1)}

This modified NLFM signal intended by means of 2 LFM function. One stage is altered by means of exponential function. MNLFM1 signal model consist NLFM slope in the first stage formed by means of exponential function and the LFM sweep in the second stage.

$f(t)=\left\{\begin{array}{lr}\alpha_{0} \exp (\alpha) t^{2} & 0 \leq t \leq T_{1} \\ B_{1}+\alpha_{1}\left(t-T_{1}\right) & T_{1} \leq t \leq\left(T_{1}+T_{2}\right)\end{array}\right.$

where $\alpha$ varies from 11 to 13 based on the T1 value. Eq. (5) characterize the MNLFM1 frequency variation with $\alpha_{0}$ and $\alpha_{1}$ being the respective slopes in the first and second stage. The phase variation given by Eq. (6) of MNLFM1 function is attained by integrating Eq. (5)

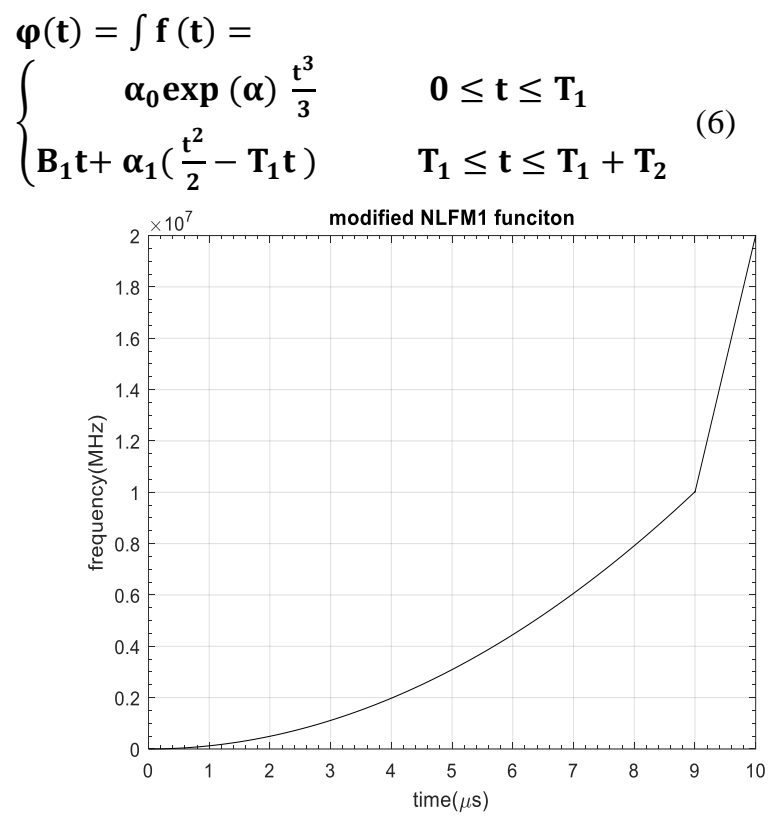

Figure.3 MNLFM1 function

\section{MODIFIED NLFM (MNLFM2)}

Second stage Modified Two-Stage signal is formed by concatenating the instantaneous frequency functions of two piece-wise functions with first stage having a LFM sweep rate of $\alpha 1$ and followed by a NLFM sweep rate of $\alpha 0$ by means of exponential function as shown in Eq. (7).

$$
\mathbf{f}(\mathbf{t})=\left\{\begin{array}{c}
\alpha_{0} \quad 0 \leq t \leq \mathrm{T}_{1} \\
\mathrm{~B}_{1}+\alpha_{1} \exp (\alpha)\left(t-\mathrm{T}_{1}\right)^{2} \mathrm{~T}_{1} \leq \mathrm{t} \leq\left(\mathrm{T}_{1}+\mathrm{T}_{2}\right)
\end{array}\right\}
$$

where $\alpha$ varies from 11 to 14 based on the T1 value. The resultant phase variation given by Eq. (8) of this concatenated NLFM function can be derived by integrating Eq. (7)

$$
\begin{aligned}
& \varphi(t)=\int f(t)= \\
& \left\{\begin{array}{cr}
\alpha_{0} \frac{t^{2}}{2} & 0 \leq t \leq T_{1} \\
B_{1} t+\alpha_{1} \exp (\alpha)\left(\frac{t^{3}}{3}-T_{1} t^{2}-T_{1}^{2} t\right) \\
T_{1} \leq t \leq T_{1}+T_{2}
\end{array}\right.
\end{aligned}
$$




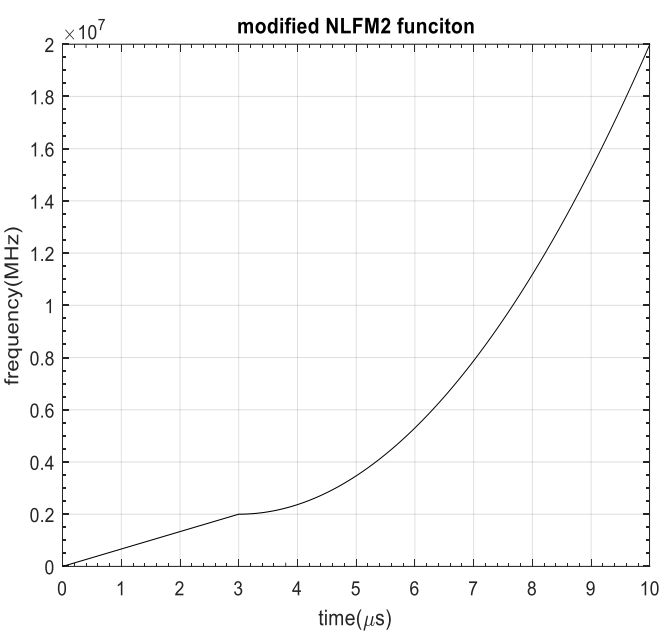

Figure.4 MNLFM2 function

\section{E. CURVED NLFM (MNLFM3)}

Curved Shaped NLFM signal is formed by concatenating the instantaneous frequency variations of two piece-wise functions with two stages having a NLFM sweep rate of $\alpha$ o and $\alpha 1$ by means of exponential functions in both the stages as shown in Eq. (9)

$$
\left\{\begin{array}{c}
\mathbf{f}(t)= \\
\boldsymbol{\alpha}_{0} \exp \left(\boldsymbol{\alpha}_{1}\right) \mathbf{t}^{2} \quad 0 \leq t \leq \mathbf{T}_{1} \\
\mathbf{B}_{1}+\boldsymbol{\alpha}_{1} \exp \left(\boldsymbol{\alpha}_{2}\right)\left(\mathrm{t}-\mathrm{T}_{1}\right)^{2} \mathrm{~T}_{1} \leq \mathrm{t} \leq\left(\mathrm{T}_{1}+\mathrm{T}_{2}\right)
\end{array}\right\}(9)
$$

where $\boldsymbol{\alpha}_{\mathbf{1}}$ varies from 11 to 13 and $\boldsymbol{\alpha}_{\mathbf{2}}$ varies from 11 to 14 based on the $\mathrm{T} 1$ value. The equivalent variation of phase Eq. (10) of this resultant NLFM function can be derived by integrating Eq. (9)

$$
\begin{aligned}
& \varphi(t)=\int f(t)= \\
& \left\{\begin{array}{cc}
\alpha_{0} \exp (\alpha) \frac{t^{3}}{3} & 0 \leq t \leq T_{1} \\
B_{1} t+\alpha_{1} \exp (\alpha)\left(\frac{t^{3}}{3}-T_{1} t^{2}-T_{1}^{2} t\right) \\
T_{1} \leq t \leq T_{1}+T_{2}
\end{array}\right.
\end{aligned}
$$

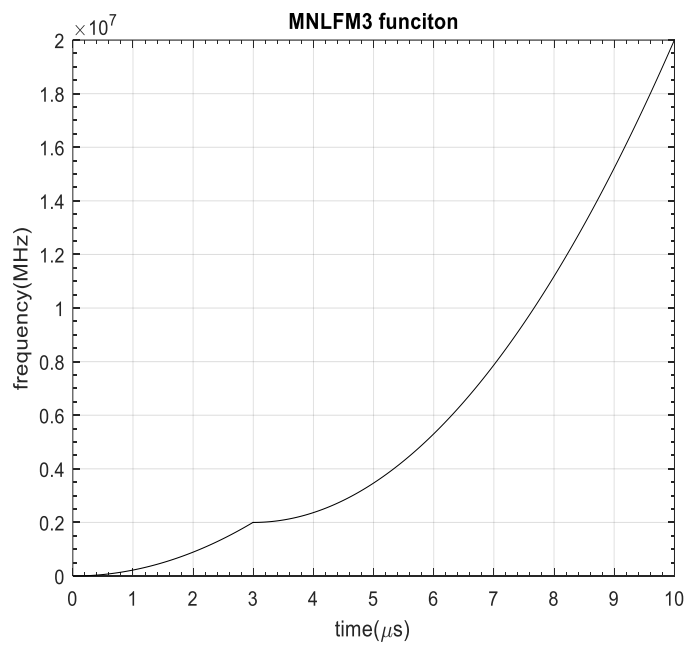

Figure.5 MNLFM3 function

Fig. 1-5 shows the frequency variation of all the designed piece-wise LFM and NLFM functions.

\section{DOPPLER EFFECT AND SIGNAL TO NOISE RATIO}

\section{A. Doppler Effect}

When a source is transmitting any kind of wave which is moving relative to the observer, a frequency shift takes place between the transmitted signal and the received signal. This effect is known as the Doppler Effect and the change in frequency is called as Doppler frequency shift or Doppler shift. The Doppler frequency shift which measures how much shift is observed in the reflected signal is calculated by the formula

$f_{d}=\frac{2 V_{r}}{\lambda}=\frac{2 V_{r} f_{c}}{c}$

where $\mathbf{f}_{\mathbf{d}}$ the Doppler shift in frequency, $\mathbf{V}_{\mathbf{r}}$ is the relative velocity of the moving target, wavelength $\lambda$ is given as $\lambda=$ $\frac{\mathbf{c}}{\mathbf{f}_{\mathrm{c}}}$, c is the speed of sound, and $\mathbf{f}_{\mathbf{c}}$ is the carrier frequency." 2 "indicate the round trip distance of the signal that is transmitted and reflecting back from the target. The amount of Doppler shift is directly proportional to velocity of moving target. In order to measure the Doppler tolerance of the NLFM and reference LFM signals, the Doppler shift frequency $\left(\mathbf{f}_{\mathbf{d}}\right)$ is calculated and added to the centre frequency $\left(\mathbf{f}_{\mathbf{c}}\right)$ of matched filter as $\left(\mathbf{f}_{\mathbf{c}} \pm \mathbf{f}_{\mathbf{d}}\right)$. The positive sign indicates the positive Doppler shift when target is moving towards the radar and the negative sign indicates the negative Doppler shift when the target is moving away from the radar [10-16].

\section{B. Background Noise}

The PSL value of NLFM waveform is greatly influenced by Doppler Effect and noise. The noise doesn't depend upon operating system frequency and has additive and Gaussian properties is Additive White Gaussian Noise (AWGN). It is a accepted noise present in all devices. The noise has constant power density and its voltage has Gaussian distribution.

\section{RESULT AND DISCUSSION}

\section{A. Doppler effect}

The simulations for all the designed NLFM waveforms are carried out for different target speeds ranging from 100 to $5000 \mathrm{~km} /$ hour. The carrier frequencies, target speeds and calculated Doppler frequencies considered for the study are shown in table 1 . The performance is compared with respect to the parameters peak side-lobe level, main lobe level and Doppler tolerance. Fig. 6 and 7 represent the ambiguity function plots of designed NLFM waveforms for $\mathbf{f}_{\mathbf{d}}=3.703$ kHz-185.185 kHz. From the Fig. 6 (a), (b) and (c) it is observed that the Two-Stage, three stage NLFM and MNLFM1 functions exhibit same Doppler characteristics as of LFM signal and for higher Doppler shifts the main lobe amplitude is reduced but still it possesses Doppler tolerance property of LFM signal. 
Performance Analysis of NLFM Signals with Doppler Effect and Background Noise

Table 1. PSLR values based on background noise

\begin{tabular}{|l|l|l|}
\hline $\mathbf{f}_{\mathbf{c}}(\mathrm{GHz})$ & $\mathbf{V}_{\mathbf{r}}(\mathrm{km} / \mathrm{hr})$ & $\mathbf{f}_{\mathbf{d}}(\mathrm{Hz})$ \\
\hline 5 & $100-5000$ & $900 \mathrm{~Hz}-47 \mathrm{kHz}$ \\
\hline 10 & $100-5000$ & $1.851 \mathrm{kHz}-92.592 \mathrm{kHz}$ \\
\hline 20 & $100-5000$ & $3.703 \mathrm{kHz}-185.185 \mathrm{kHz}$ \\
\hline 30 & $100-5000$ & $1.851 \mathrm{kHz}-277.777 \mathrm{kHz}$ \\
\hline
\end{tabular}

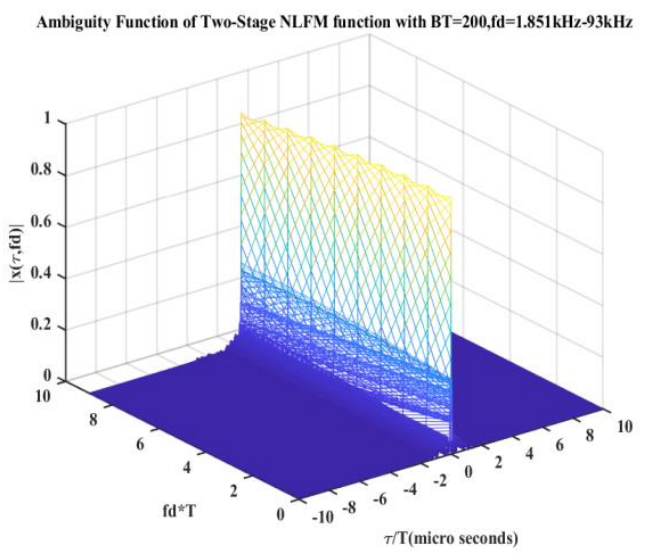

Ambiguity Function of Three-stage NLFM function with BT=200,fd=1.851kHz-93kHz

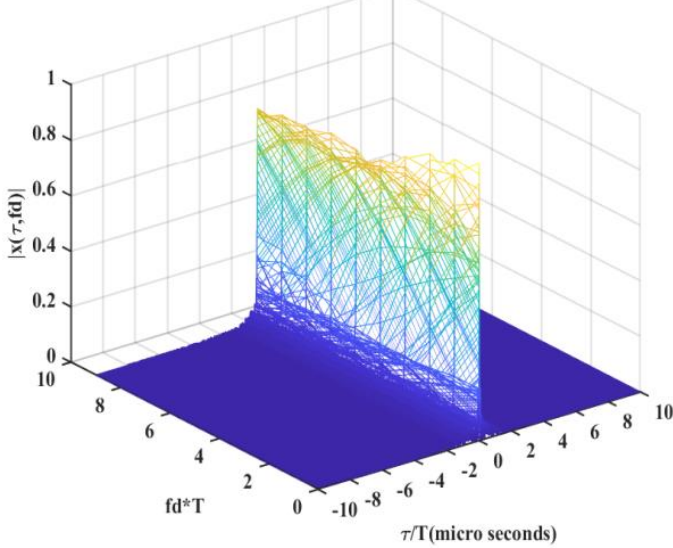

Ambiguity Function of MNLFM1 function with $\mathrm{BT}=300, \mathrm{fd}=1.851 \mathrm{kHz}-93 \mathrm{kHz}$

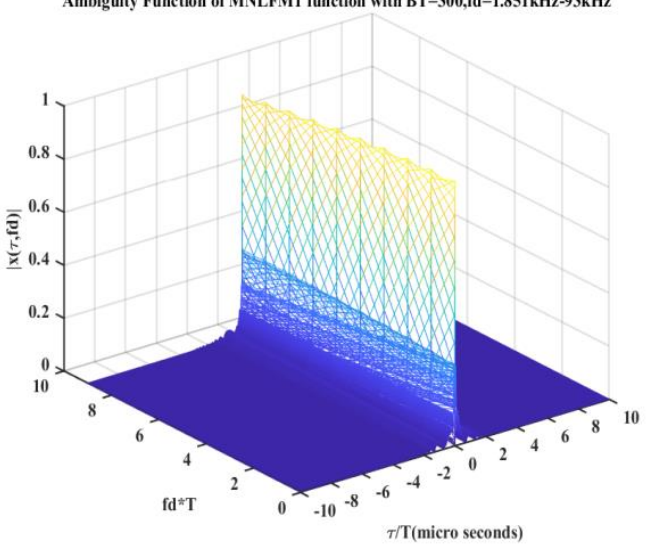

Figure.6 Ambiguity functions for $f_{d}=3.703 \mathrm{kHz}-185.185$ kHz of: (a) NLFM1 and (b) NLFM2 (c) MNLFM1

The ambiguity function plots of MNLFM2 and MNLFM3 are shown in Fig. 7 (a) and (b) respectively. Both the signals exhibited same characteristics as that of LFM signal when the Doppler frequency is in the range of $900 \mathrm{~Hz}-47 \mathrm{kHz}$.

Retrieval Number: B3835129219/2020@BEIESP

DOI: 10.35940/ijeat.B3835.029320

Journal Website: www.ijeat.org
Whereas when the Doppler frequency is in the range of 1.85 $\mathrm{kHz}-93 \mathrm{kHz}$ the main lobe level amplitude decreased but still exhibits Doppler tolerance.

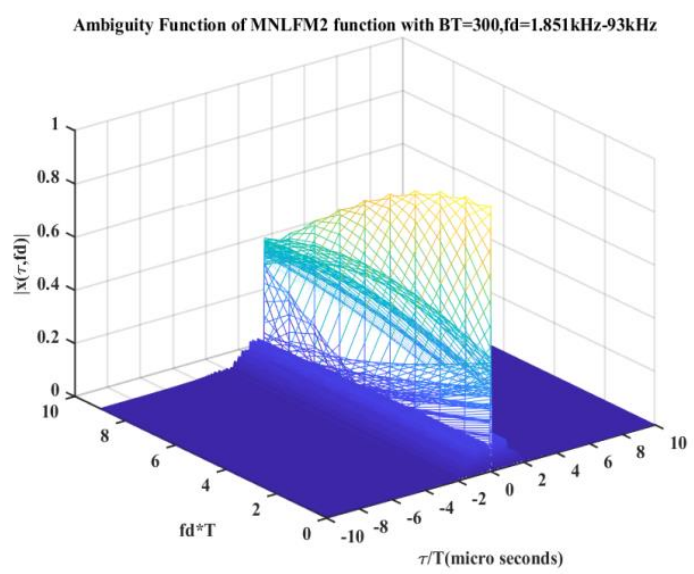

Figure.7 Ambiguity functions for $f_{d}=3.703 \mathrm{kHz}-185.185$ kHz of: (a) MNLFM3 and (b) MNLFM3

The Doppler Effect to peak side-lobe level and main lobe level of designed waveforms are shown in Fig.8 and 9.

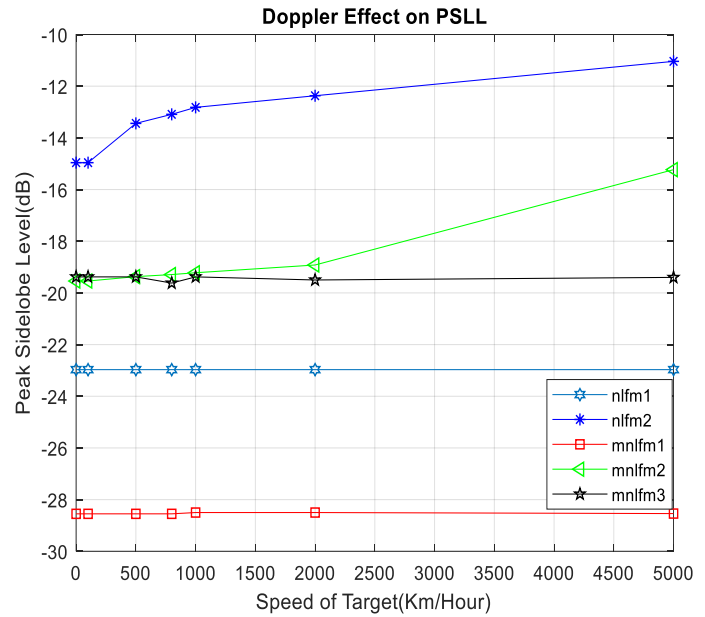

Figure.8 Doppler Effect to PSLR values of NLFM Signals

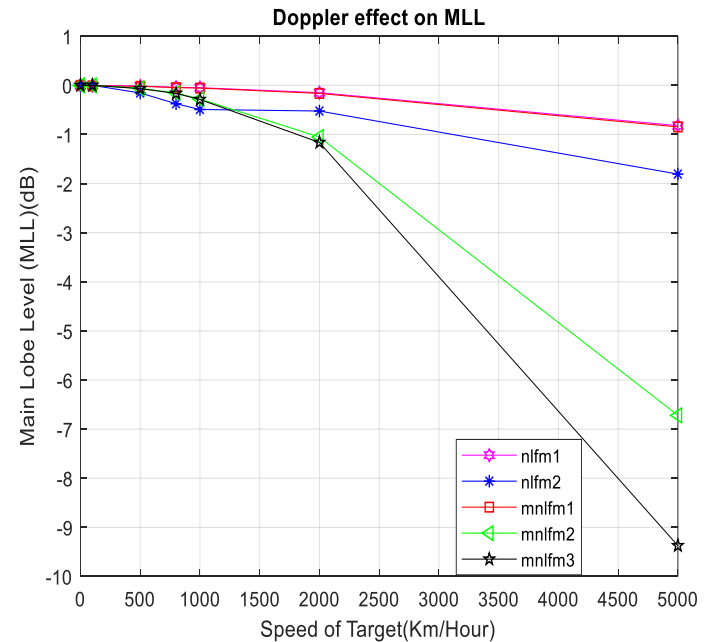

Figure.9 Doppler Effect to MLL values of NLFM signals. 
From the simulations we illustrate that the designed NLFM1, NLFM2, NLFM3 and MNLFM1 signals exhibit same Ambiguity function characteristics as of LFM signal and are Doppler tolerant. MNLFM2 and MNLFM3 is Doppler intolerant to higher Doppler frequency shifts. PSLR values of all the signals are affected with the change in the speed of the target. MNLFM1 exhibits highest PSLR value amongst all other signals with the change in the speed of the target. Main Lobe Level amplitude is also highly affected with the change in the speed of the target. MLL values of MNLFM2 and MNLFM3 signals are drastically reduced with the increase in the speed of the target.

\section{B. Background Noise}

To study the effect of AWGN on the designed NLFM waveforms the noise is added to the signals with SNR ranging from $20 \mathrm{~dB}$ to $-20 \mathrm{~dB}$. The measurement is done by taking several trials and noting the mean value obtained. PSLR values obtained for different waveforms are tabulated in Table 2. For SNR of $20 \mathrm{~dB}$, the PSLR value of NLFM two stage and three stage is around -34.84 and -34.70 respectively. For -20dB SNR the PSLR values are around $16.02 \mathrm{~dB}$ and $-16.75 \mathrm{~dB}$ respectively. The Values in both the cases are almost similar. Whereas for modified NLFM waveforms the PSLR values at $20 \mathrm{~dB}$ SNR are around 29.44, -34.43 and -29.38 respectively. When SNR dropped to $-20 \mathrm{~dB}$ the PSLR values for these waveforms are 15.83dB, $-13.56 \mathrm{~dB}$ and $-14.5 \mathrm{~dB}$ respectively. From Fig. 10 as the SNR changes from -20 to $20 \mathrm{~dB}$ the PSLR values of all waveforms exhibited the same pattern. The increase in SNR value caused the PSLR to increase. The main lobe parameters are not affected. Among the waveforms, the PSL of NLFM waveform designed with two piece-wise LFM is around $-34.84 \mathrm{~dB}$.

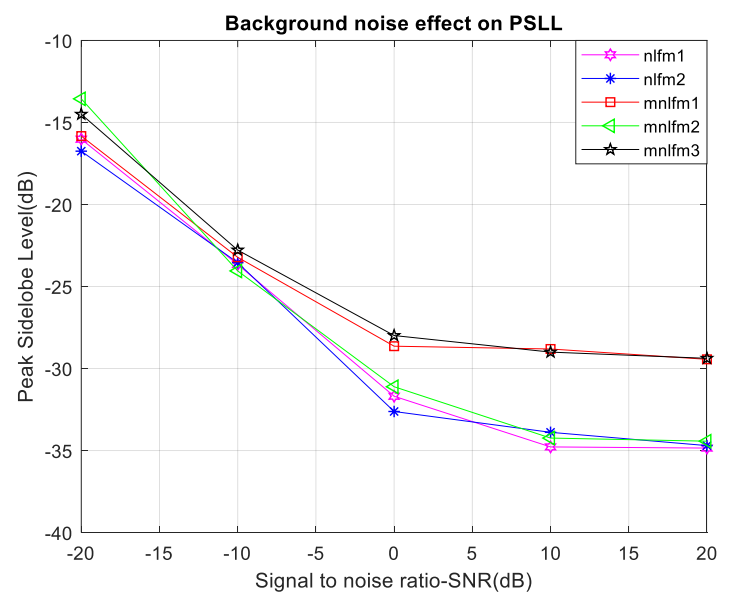

Figure.10 Background noise effect on PSLR values

Table 2. PSLR values based on background noise

\begin{tabular}{|l|l|l|l|l|l|}
\hline $\begin{array}{l}\text { SNR } \\
(\mathrm{dB})\end{array}$ & \multicolumn{5}{|l}{ PSLR (dB) } \\
\hline & NLFM1 & NLFM2 & $\begin{array}{l}\text { MNLF } \\
\text { M1 }\end{array}$ & $\begin{array}{l}\text { MNLF } \\
\text { M2 }\end{array}$ & $\begin{array}{l}\text { MNLF } \\
\text { M3 }\end{array}$ \\
\hline-20 & -16.02 & -16.75 & -15.83 & -13.56 & -14.5 \\
\hline-10 & -23.66 & -23.55 & -23.22 & -24.05 & -22.78 \\
\hline 0 & -31.69 & -32.62 & -28.64 & -31.11 & -27.99 \\
\hline 10 & -34.78 & -33.89 & -28.8 & -34.24 & -29 \\
\hline 20 & -34.84 & -34.70 & -29.44 & -34.43 & -29.38 \\
\hline
\end{tabular}

From the simulations it is evident that the lower SNR value causes decrease in PSL while the main lobe parameters remain unaffected. However PSLR value increased and main lobe level decreased with change in speed of the target. The NLFM function designed using two piece-wise LFM is Doppler tolerant and as the SNR changes from -20 to $20 \mathrm{~dB}$ the PSL of this signal is around $-34.84 \mathrm{~dB}$. Hence, the NLFM designed with two piece-wise LFM proved to be better than NLFM designed waveforms for detectability and resolution in radar applications

\section{REFERENCES}

1. Skolnik. M.I, Radar Handbook, 3rd Edition, USA: McGraw-HILL, 2008.

2. Mozeson. E and Levanon. N, Radar Signals. Wiley New York, 2004. S. Boukeffa, Y. Jiang and T. Jiang, "Sidelobe reduction with nonlinear frequency modulated waveforms," 2011 IEEE 7th International Colloquium on Signal Processing and its Applications, Penang, 2011, pp. 399-403.

3. I. Vizitiu, L. Anton, F. Popescu and G. Iubu, "Sidelobes reduction using frequency predistortioning techniques on LFM signals," 2012 10th International Symposium on Electronics and Telecommunications, Timisoara, 2012, pp. 381-384.

4. I. Vizitiu, L. Anton, F. Popescu and G. Iubu, "The synthesis of some NLFM laws using the stationary phase principle," 2012 10th International Symposium on Electronics and Telecommunications, Timisoara, 2012, pp. 377-380.

5. W. Yue and Y. Zhang, "A novel nonlinear frequency modulation waveform design aimed at side-lobe reduction," 2014 IEEE International Conference on Signal Processing, Communications and Computing (ICSPCC), Guilin, 2014, pp. 613-618.

6. C.E.Cook and J. Paolillo, "A pulse compression predistortion function for efficient sidelobe reduction in a high-power radar," in Proceedings of the IEEE, vol. 52, no. 4, pp. 377-389,April,1964.

7. H. D. Griffiths and L. Vinagre, "Design of low-sidelobe pulse compression waveforms," Electron. Lett. vol. 30, no. 12, Jun. 1994.

8. Arif. M, et al, Investigation of Non-linear Chirp Coding for Improved Second Harmonic Pulse Compression. Ultrasound in Medicine \& Biology, 43 (8). pp. 1690-1702. ISSN 0301-5629

9. Nettem. Adithya valli, Daniel. Elizabath Rani, Chandu. Kavitha, "Doppler Effect Analysis of NLFM signals". International journal of scientific \& technology research (IJSTR), Volume-8, Issue-11, November 2019.

10. Adithya valli. N, Elizabath Rani. D, "Modified PWNLFM Signal for Side-Lobe Reduction". International Journal of Engineering \& Technology, v.7, n.4.20, p.4-7, Nov.2018.

11. N. Adithya valli, D. Elizabath Rani, C. Kavitha, "An Algorithm for Computing Side-lobe Values of a Designed NLFM function". International Journal of Advanced Trends in Computer Science and Engineering, v.8, n.4, August 2019. Doi https://doi.org/10.30534/ijatcse/2019/07842019

12. N. Adithya valli, D. Elizabath Rani, C. Kavitha, "Windows for reduction of ACF sidelobes of pseudo-NLFM signal". International Journal of Scientific \& Technology Research (IJSTR), Volume-8, Issue-10, October 2019

13. N. Adithya valli, D. Elizabath Rani, C. Kavitha, "Modified Radar Signal Model using NLFM". International Journal of Recent Technology and Engineering (IJRTE), Volume-8, Issue-2S3, July 2019.

14. N. Adithya valli, D. Elizabath Rani, C. Kavitha, "Convolutional Windows for Side-lobe Reduction". International Journal of Innovative Technology and Exploring Engineering (IJITEE), Volume-8 Issue-10, August 2019.

15. N. Adithya valli, D. Elizabath Rani, C. Kavitha, "Conventional vs Convolutional Windows for Reduction of Side-lobes". International Journal of Engineering and Advanced Technology (IJEAT), Volume8 Issue-6, August2019. 


\section{AUTHORS PROFILE}

N. Adithya valli, was born in 1988, she completed her under-graduate studies in electronics and communications engineering (ECE) at Gudlavaleru engineering college (affiliated to jntu Kakinada), Vijayawada (India) in 2010 and she received her dual degree (M.Tech/m.sc) in electrical engineering with emphasis on signal processing at jntu Kakinada and Blekinge institute of technology (BTH) Sweden in 2013. She worked as assistant professor in Lingaya's engineering college Vijayawada (India) from 2014 to 2015. Now she is pursuing her full time $\mathrm{PhD}$ in Gitam University Visakhapatnam.

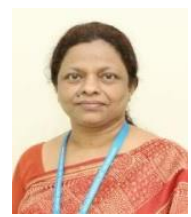

D. Elizabath rani, received the $\mathrm{PhD}$ degree in the field of Radar Signal Processing from Andhra University in the year 2003. Presently, she is Professor and in charge Principal of GITAM Institute of Technology, GITAM University, Visakhapatnam, India .Her research interests are Signal processing techniques in Radar, Sonar and Bio-Medical field. She is a member of IETE and ISTE. She has more than 40 papers published to her credit in reputed international/national journals and conferences.

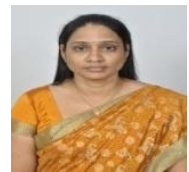

C. Kavitha, Associate Professor, Department of Physics, Institute of Technology, GITAM (deemed to be University) has more than twenty two years of service in academics. Her interests are in the fields of embedded systems and IoT. She has authored more than twenty reviewed research articles and four books. 